\title{
Innovative Strategies for the Removal of Organic Compounds in Nanoparticle ITO Layers
}

\author{
Florian Mikschl $^{* 1,2}$, Marcus Baum ${ }^{* 1,2}$ and Michael Schmidt ${ }^{* 1,2}$ \\ ${ }^{* 1}$ Institute of Photonic Technologies (LPT), Friedrich-Alexander Universität Erlangen-Nürnberg, \\ Konrad-Zuse-Straße 3/5, 91052 Erlangen, Germany \\ E-mail: florian.mikschl@lpt.uni-erlangen.de \\ ${ }^{*} 2$ Erlangen Graduate School in Advanced Optical Technologies (SAOT), Paul-Gordan-Straße 6, \\ 91052 Erlangen, Germany
}

\begin{abstract}
Layers of indium-tin-oxide (ITO) nanoparticles were deposited on the polymer substrates polypropylene and polyimide in ambient air. To decrease the electrical resistance of the deposited nanoparticle ITO layer precipitated organic compounds of the nanoink have to be removed. For temperature-resistant substrates a baking step is suitable for the removal of organic agents utilizing a hotplate. However, a hotplate baking is not suitable for organic substrates. In order to avoid thermal damage the polymer substrate laser based removal strategies were investigated utilizing ultraviolet and infrared laser irradiation. To further optimize the electrical conductivity of the ITO nanoparticle layer a final laser consolidation step is essential utilizing ultraviolet laser light provided by a $\mathrm{KrF}$ excimer laser.
\end{abstract}

DOI: $10.2961 / \mathrm{jlmn} .2016 .01 .0023$

Keywords: ITO, nanoparticles, laser, removal, organic components

\section{Introduction}

ITO provides functionality in many optoelectronic applications such as displays and solar cells because it combines optical transparency in the visible light spectrum with high electrical conductivity. Commonly ITO layers are generated by vacuum deposition techniques, like sputtering and chemical vapor deposition [1-3]. Vacuum based deposition techniques are cost intensive and lack from the flexibility required for modern production techniques, like rollto-roll processing. The upcoming nanotechnology gave rise to a myriad of novel approaches of ITO layer generation in ambient air. Thereby nanoinks with ITO nanoparticles are used, which enable inexpensive production techniques in ambient air, like gravure printing or inkjet printing. Here structures of ITO are generated by precipitation of nanoparticles from the liquid phase $[4,5]$. However, the electrical resistance of layers, generated by deposition techniques in ambient air, is much higher than the electrical resistance of layers, generated by vacuum deposition techniques $[6,7]$. Layers of ITO with a sheet resistance of $20 \Omega$ or less can be realized by vacuum deposition, whereas the sheet resistance of ITO layers deposited in ambient air is at least one order of magnitude greater than the sheet resistance of ITO layers generated by vacuum deposition $[8,9]$. Especially for power electronics a low sheet resistances are essential, for instance $4 \Omega$ for a planar microwave antenna or $10 \Omega$ for a solar cell $[10,11]$. To overcome this limitation we developed a novel consolidation approach utilizing single pulse ultraviolet laser irradiation in ambient air. The laser pulse $(248 \mathrm{~nm})$ is strongly absorbed by the ITO particles due to interband interaction which leads to significant heating of the ITO layer surface. Particle melting and diffusion effects then cause a significant densification of the particle layer $[6,12,13]$. This laser consolidation procedure has proven valid for glass substrates. But this ap- proach requires a preliminary hotplate treatment $\left(450^{\circ} \mathrm{C}\right.$, $30 \mathrm{~min}$ ) to eliminate organic agents covering the surface of the deposited ITO nanoparticles. Otherwise the organic components in the deposited ITO layer are decomposed by the UV irradiation. The rapidly expanding decomposition products then damage the surrounding nanoparticle layer. For a number of electronical applications organic substrates have considerable advantages compared to glass substrates. For instance a superior mechanical flexibility of the polymer substrates offers applications like flexible displays or bendable solar cells. Lower costs of polymer substrates compared to glass enable the production of inexpensive transparent electronic and disposals. As mentioned before the laser consolidation process requires a temperature of $450{ }^{\circ} \mathrm{C}$, which exceeds the decomposition temperature of most organic substrates. Consequently the thermal removal of organic substrates from deposited ITO layers is not suitable for organic substrates utilizing hotplate removal. In order to use the superior features of organic substrates for consolidated nanoparticle ITO layers methods for the removal of the organic compounds have to be investigated. In the following we present approaches for the laser based removal of organic compounds in the deposited ITO particle layer focusing on ultraviolet and infrared laser irradiation.

\section{Experimental details}

In the following paragraph we describe the ITO layer generation, the various subsequent removal approaches (hotplate and laser based) and the final laser consolidation in detail. 


\subsection{Layer generation}

In our experiments we used ITO nanoparticles commercially available by Evonik Industries AG. The size distribution of the particles was ranging from $20 \mathrm{~nm}$ to $120 \mathrm{~nm}$. The particles were dispersed in absolute alcohol at a weight loading of $20 \%$. Agglomerations of the ITO nanoparticles were prevented by addition of the organic agent 2-[2-(2-methoxy-ethoxy)ethoxy]acetic acid which prohibits agglomeration of the particles due to steric hindrance [14].

The nanoparticle layers were deposited on three different substrate materials via spin coating at $3000 \mathrm{rpm}$ for $20 \mathrm{~s}$. The first two substrates were organic polymer foils. Polyimide (PI) (Du Pont), with a thickness of $127 \mu \mathrm{m}$ and a polypropylene (PP) substrate (Esselte 54820) with a thickness of $110 \mu \mathrm{m}$ was used. The PI substrate was chosen because PI is used in a wide range of electronic applications, such as in motor and drive technology or aerospace. PI offers superior thermal properties and was chosen in respect of the class of high-temperature polymers. In contrast the thermoplastic PP substrate provides inferior thermal characteristics and represents the majority of polymers which are temperature-sensitive. The glass transition temperature of PI is at approximately $360{ }^{\circ} \mathrm{C}$, whereas the melting range of $\mathrm{PP}$ is at approximately $160^{\circ} \mathrm{C}$. These polymers were chosen to evaluate the laser based removal processes for the groups of inexpensive temperaturesensitive substrates and temperature resistant substrates. For handling issues the organic substrates were glued on a soda lime glass substrate $(25 \mathrm{~mm}$ x $25 \mathrm{~mm}$ x $1 \mathrm{~mm})$ utilizing a two component adhesive (UHU plus endfest 300). As a reference we used soda lime glass substrate. ITO layers were generated on soda lime glass to be able to compare the novel laser based removal techniques with the state of the art hotplate removal.

To determine the thickness of the deposited nanoparticle ITO layer scratches were introduced on the ITO layer and the heights profile was measured by a laser scanning microscope (LSM) (Olympus Lext OLS4000). ITO layer thicknesses of approximately $465 \mathrm{~nm}$ were achieved on the glass substrate and thicknesses of approximately $475 \mathrm{~nm}$ on the PI substrate. We were not able to determine the layer thickness for the PP substrate, because the scratching process deformed the nanoparticle layer and the PP substrate beneath.

\subsection{Removal of organic compounds}

The organic agent TODA generates a micelle around the ITO nanoparticle and therefore prohibits the nanoparticles from agglomeration due to steric hindrance. Consequently the organic shells of the nanoparticles are also deposited generating the nanoparticle ITO layer. To decrease the electrical resistance of the deposited ITO layer the organic compounds have to be removed in order to enhance the particle-particle-contact. To achieve this goal a baking of the deposited nanoparticle ITO layers is required utilizing a hotplate.

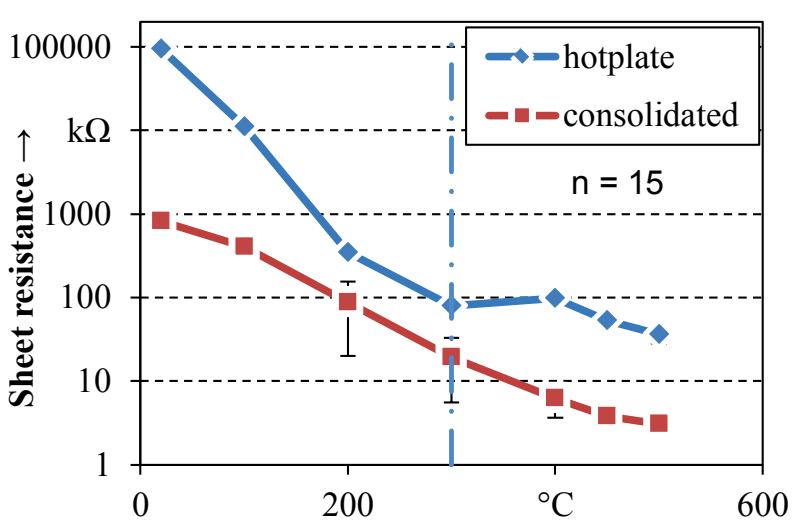

Hotplate removal temperature $\rightarrow$

Fig. 1 Sheet resistance of post-baked nanoparticle ITO layers (hotplate) and sheet resistance of laser consolidated nanoparticle ITO layers (consolidated) deposited on soda lime glass substrates. The baking time was $30 \mathrm{~min}$.

Figure 1 shows the sheet resistance of a post-baked nanoparticle ITO layer depending on the temperature of the hotplate. For the measurements of the sheet resistance a 34410A digital multimeter (Agilent) was used in four probe configuration. ITO layers were deposited on soda lime glass. Then the layers were heated up and after the baking the sheet resistance of the layer was characterized. These values are named "hotplate" in figure 1 and figure 2 . The sheet resistance of layers, which were consolidated after the baking are named "consolidated" in figure 1 and figure 2. It is shown that a temperature of $300{ }^{\circ} \mathrm{C}$ is sufficient for the total removal of organic compounds. The sheet resistance of the ITO layer after the baking step (post-baked) is decreasing till a hotplate temperature of $300{ }^{\circ} \mathrm{C}$, because the organic compounds are removed from the deposited layer. From $300{ }^{\circ} \mathrm{C}$ to $400{ }^{\circ} \mathrm{C}$ the sheet resistance is approximately constant. We assume all organic compounds are removed at this temperature range. Removal temperatures over $400{ }^{\circ} \mathrm{C}$ induce a further decrease of the sheet resistance due to thermal annealing, diffusion processes or probably by crystallization. The dependency of the baking duration on the sheet resistance of post-baked ITO layers is shown in figure 2. The duration of the hotplate process has a less significant effect on the sheet resistance of postbaked nanoparticle ITO layers. As a tendency higher baking durations decrease the sheet resistance of the ITO layers due to diffusion and annealing except for the sheet resistance measured after a removal duration of $10 \mathrm{~min}$ which is most likely an outlier indicated by a vast standard deviation. Consequently hotplate removal is not suitable for organic substrates, because a temperature of at least $300^{\circ} \mathrm{C}$ has to be applied for at least several minutes. But the high thermal capacity of organic substrates would allow a temporary heating of the ITO layer above of the substrate without damaging it. 


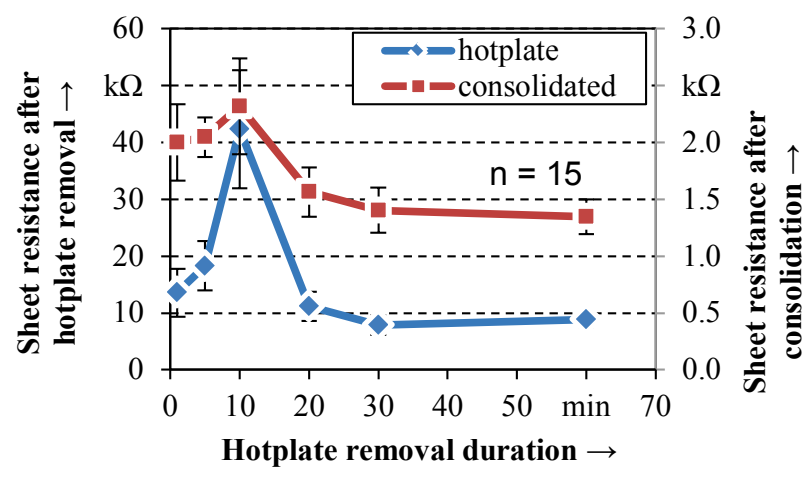

Fig. 2 Sheet resistance of post-baked nanoparticle ITO layers (hotplate) and laser consolidated ITO layers (consolidated) on soda lime glass substrates. The baking temperature was $450{ }^{\circ} \mathrm{C}$.

For the removal of organic agents in the deposited ITO layers two laser based removal treatments were investigated. First a $\mathrm{CO}_{2}$ laser (Diamond $\mathrm{K} 150$, Coherent) was used with a wavelength of $10.6 \mu \mathrm{m}$, a maximal average power of $150 \mathrm{~W}$, a maximal pulse frequency of $100 \mathrm{kHz}$ and a maximal pulse length of $1 \mathrm{~ms}$. The laser beam was deflected by a galvanometer scanner (Scangine 10, SCANLAB AG) with a working distance of $137 \mathrm{~mm}$. The focal length of the f-theta lens was $150 \mathrm{~mm}$. In the focus the beam diameter is approximately $800 \mu \mathrm{m}$. Second a $\mathrm{KrF}$ eximer laser (LPX 315i, Lambda Physik) was utilized with a wavelength of $248 \mathrm{~nm}$, a maximal pulse energy of $0.8 \mathrm{~J}$, pulse length of $30 \mathrm{~ns}$ and a raw beam diameter of $30 \mathrm{~mm} \times 15 \mathrm{~mm}$. To adjust the fluence we used a lens with a focal length of $200 \mathrm{~mm}$. An aperture $(30 \mathrm{~mm} \times 5 \mathrm{~mm})$ was placed at a distance of $200 \mathrm{~mm}$ before the sample to cut off the inhomogeneous borders of the laser beam. For referencing organic compounds were removed from coated soda lime glass substrates by thermal removal. For this approach a hotplate was used and the layers were annealed at a temperature of $450{ }^{\circ} \mathrm{C}$ for $30 \mathrm{~min}$.

\subsection{Consolidation of the nanoparticle ITO layers}
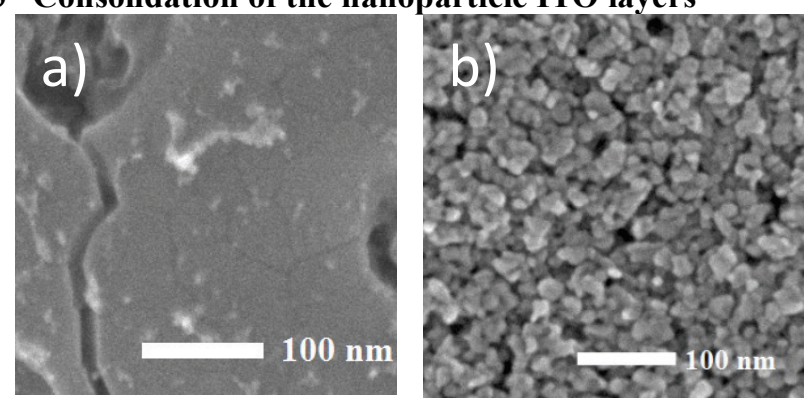

Fig. 3 a) Top view of a SEM image of a consolidated ITO layer (consolidation fluence of $54.1 \mathrm{~mJ} / \mathrm{cm}^{2}$ ); b) top view of a SEM image of a spin coated layer.

For the consolidation of the nanoparticle ITO layers single pulse ultraviolet laser irradiation was used utilizing a $\mathrm{KrF}$ excimer laser (LPX 315i, Lambda Physik) with a wavelength of $248 \mathrm{~nm}$, a maximal pulse energy of $0.8 \mathrm{~J}$, pulse length of $30 \mathrm{~ns}$, a repetition rate of $100 \mathrm{~Hz}$ and a raw beam diameter of $30 \mathrm{~mm}$ x $15 \mathrm{~mm}$. The raw beam was limited to $30 \mathrm{~mm} \times 5 \mathrm{~mm}$ by an aperture to cut of the inhomogeneous regions at the borders of the raw beam.
The surface of a consolidated and a deposited nanoparticle ITO layer is shown in figure 3. The laser consolidation densifies approximately $100 \mathrm{~nm}$ of nanoparticle layer starting from the top of the layer and reduces the sheet resistance of from the $\mathrm{M} \Omega$-range for the deposited layer to approximately $600 \Omega$ with ideal conditions due to the densification of layer surface [6]. For the laser consolidation approach the preceding and complete removal of organic compounds in the nanoparticle ITO layer is essential. Otherwise the organic compounds are decomposed instantaneously by the laser irradiation of the $\mathrm{KrF}$ excimer laser and the reaction products impair the surrounding ITO layer. This trend can be seen in figure 1 and figure 2. Figure 1 and figure 2 also show that the resistance of the ITO after laser consolidation is also depending on the electrical resistance of the particle layer. After consolidating the sheet resistance of the consolidated nanoparticle ITO layer equals a parallel connection of two resistors. The first resistor is the densified part of the nanoparticle ITO layer and the remaining particle layer (after the laser consolidation approach) equals the second resistor. Consequently, thermal annealing also has an effect on the sheet resistance of the consolidated layer due to the parallel connection of resistances. This behavior is shown in fig. 1 . In this case electrical conductivity of the particle layer beneath the consolidated layer is also increased and therefore the electrical conductivity of the total layer is increased. Figure 4 illustrates the optical transparency of consolidated ITO layers on soda lime glass substrates. In the visual wavelength range (400 $\mathrm{nm}$ to $800 \mathrm{~nm}$ ) ITO shows an average transmission of approximately $85 \%$. However, utilizing higher fluences for the consolidation of nanoparticle ITO layers decreases the optical transmission of the ITO layer. Increasing consolidation fluences lead to a rising amount of cracks and ablations which act as scattering centers and therefore reduce the optical transmission [6].

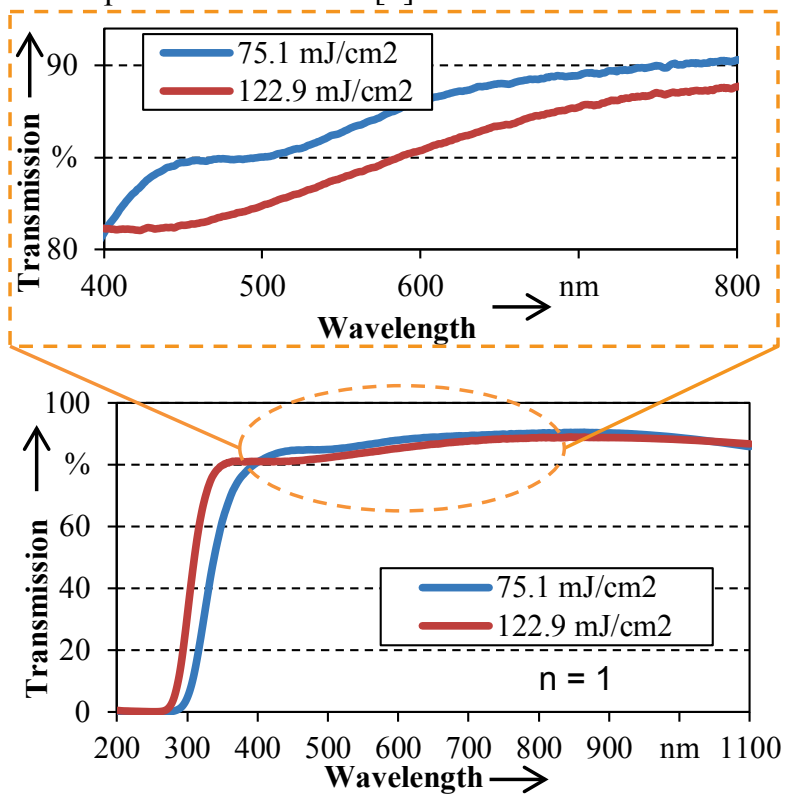

Fig. 4 Optical transmission of ITO nanoparticle layers consolidated (fluence: $75.1 \mathrm{~mJ} / \mathrm{cm}^{2}$ and $122.9 \mathrm{~mJ} / \mathrm{cm}^{2}$ ) on soda lime glass. The ITO layer thickness was approximately $530 \mathrm{~nm}$. 


\section{Results and discussion}

\section{1 $\mathrm{CO}_{2}$ laser treatment}

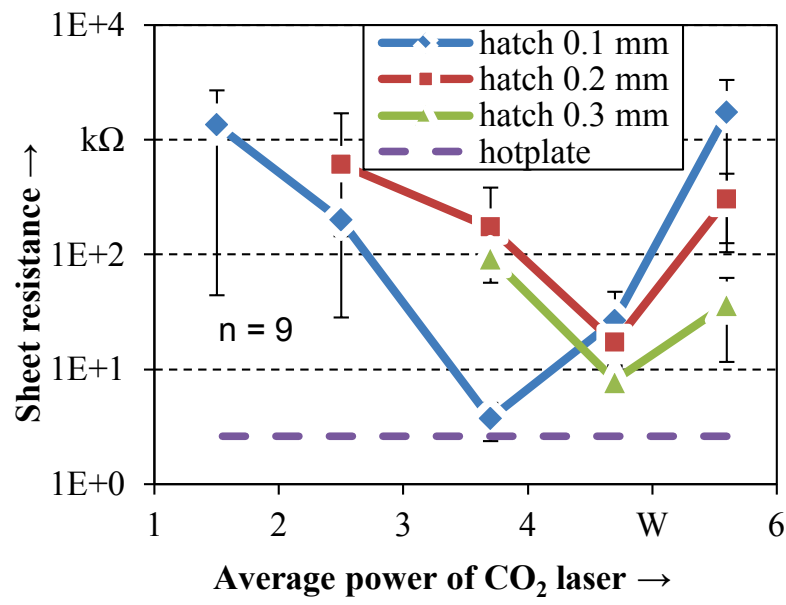

Fig. 5 Sheet resistance of consolidated ITO layers on PI substrate (fluence of approximately $46.7 \mathrm{~mJ} / \mathrm{cm}^{2}$ ).

For the $\mathrm{CO}_{2}$ laser based removal a pulse frequency of $1 \mathrm{kHz}$ and a pulse period of $50 \mu \mathrm{s}$ was applied. The feed rate was adjusted to $25 \mathrm{~mm} / \mathrm{s}$. The pulse period and the feed rate enable a high selective heat input into the ITO layer, whereas the relatively low pulse frequency was chosen to ensure a thermal relaxation of the ITO layer between the pulses. At first the optimal spot diameter and thereby the optimal energy density was identified. For ITO layers on a glass substrate a working distance of $170 \mathrm{~mm}$ has shown the best results. The working distance is defined as the distance from the exit aperture of the galvanometer scanner to the workpiece. At this point the electrical properties of these ITO layers are comparable to the electrical properties of layers treated by hotplate baking. Together with the laser conditions the hatch and the distance control the heat input and the peak temperature in the ITO composite structure. The hatch is the center-to-center distance of two following passes of the laser beam. Distances less than $170 \mathrm{~mm}$ led to ablations of the ITO particle layer or to thermal impairment of the glass substrate. The thermal removal of the organic compounds at a distance greater than $170 \mathrm{~mm}$ was not complete. The optimal hatch at the distance of $170 \mathrm{~mm}$ was $0.1 \mathrm{~mm}$. The $1 / \mathrm{e}^{2}$ beam diameter was $8.4 \mathrm{~mm}$ at this distance.

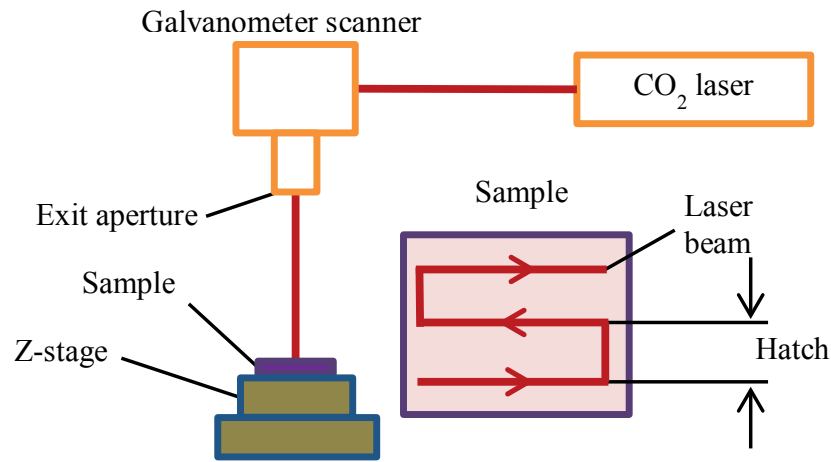

Fig. 6 Experimental setup of the removal of organic agents in nanoparticle ITO layers utilizing $\mathrm{CO}_{2}$ laser irradiation.
The experimental setup is shown in figure 6 . The hatch was varied to optimize the temperature distribution during the laser treatment. The optimal working distance of $170 \mathrm{~mm}$ was confirmed for PI substrates. Figure 5 shows the sheet resistances of consolidated ITO layers after $\mathrm{CO}_{2}$ laser based removal of the organic compounds. It is shown that an average laser power of $3.7 \mathrm{~W}$ and a hatch distance of $0.1 \mathrm{~mm}$. With these parameters a total removal of the organic agents is possible. Consequently, the total ITO nanoparticle layer has to be exposed to a temperature of at least $300{ }^{\circ} \mathrm{C}$. A thermal impairment of the organic substrate could be avoided due to the fast heating and cooling rates laser irradiation supplies. With this parameters sheet resistances of consolidated ITO layers were realized which are only 1.4 times of the mean sheet resistance of the glass reference substrates.
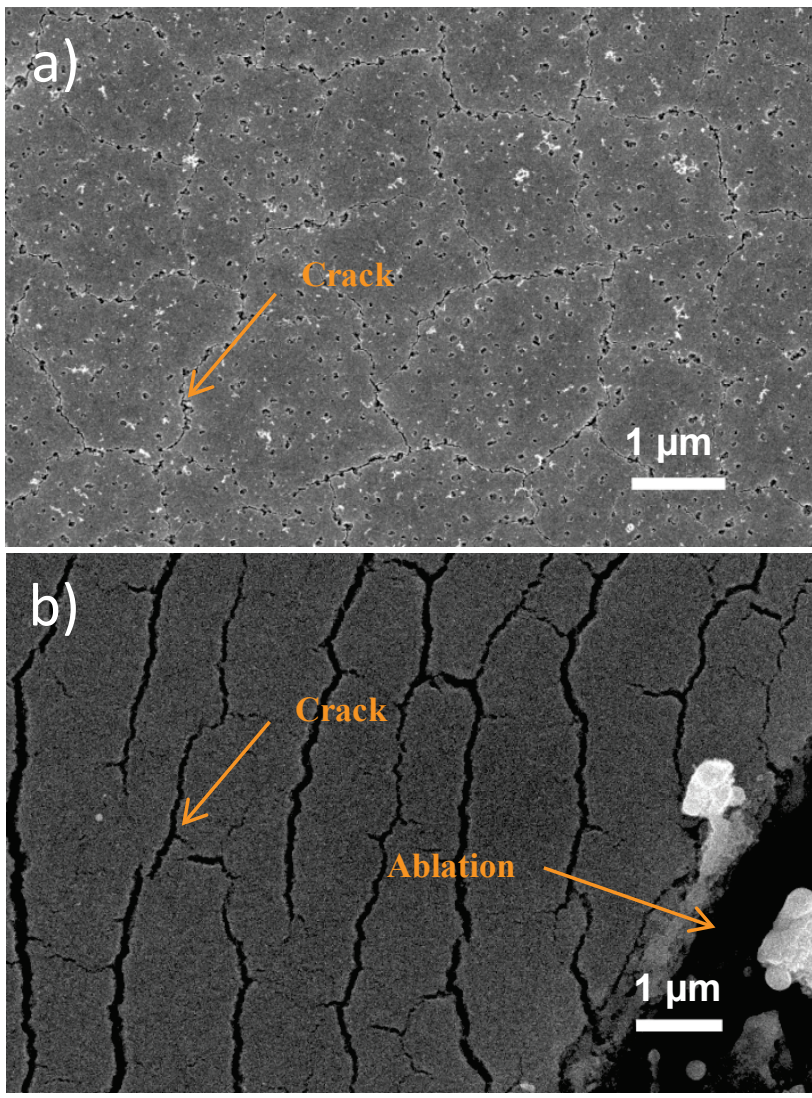

Fig. 7 Top view of a SEM image of a consolidated ITO layer (consolidation fluence of approximately $46.7 \mathrm{~mJ} / \mathrm{cm}^{2}$ ) on a) a soda lime glass substrate and b) a PI substrate.

The major influence to the electrical resistance of consolidated ITO layers is the homogeneity of the densified nanoparticle layer [6]. In other words consolidated ITO layers with a homogeneous surface show higher electrical conductivity than layers with distinct surface defects. Figure 7 shows the surfaces of consolidated ITO layers deposited on a glass and on a PI substrate. The surface of the consolidated ITO layer deposited on the PI substrate shows a distinct defect structure, compared to the consolidated ITO layer deposited on the glass substrate. This damage is most likely caused by mechanical deformation, introduced by thermal expansion of the multilayer system during the $\mathrm{CO}_{2}$ or $\mathrm{KrF}$ laser irradiation $[15,16]$. Figure 8 shows the top view of an ITO layer deposited on PI before laser irradiation and two 
ITO layer after excimer laser consolidation. Figure $8 \mathrm{~b}$ shows the top view of a consolidated ITO layer after $\mathrm{CO}_{2}$ laser irradiation with ideal parameters, whereas figure 8a shows the top view of a consolidated ITO layer after incomplete $\mathrm{CO}_{2}$ laser based removal of organic agents. The infrared $\mathrm{CO}_{2}$ laser irradiation was not applicable for the PP substrate. $\mathrm{CO}_{2}$ laser irradiation leads to impairment of the PP substrate and due to the fact to an ablation of the ITO layer. The laser induced damage of the PP surface after $\mathrm{CO}_{2}$ laser irradiation can be seen in figure 8c. The transmission of the nanoparticle ITO layer with a thickness of circa $416 \mathrm{~nm}$ is approximately $70 \%$ at the wavelength of $10.6 \mu \mathrm{m}$. Consequently a substantial amount of laser energy is deposited in the multilayer system below and heats up the system. For a sufficient removal of organic compounds from the deposited ITO layer a temperature of approximately $300{ }^{\circ} \mathrm{C}$ is required. Consequently the thermal heat load deposited in the multilayer system damages the PP substrate severely and as a result the ITO particle layer is removed from the PP substrate.

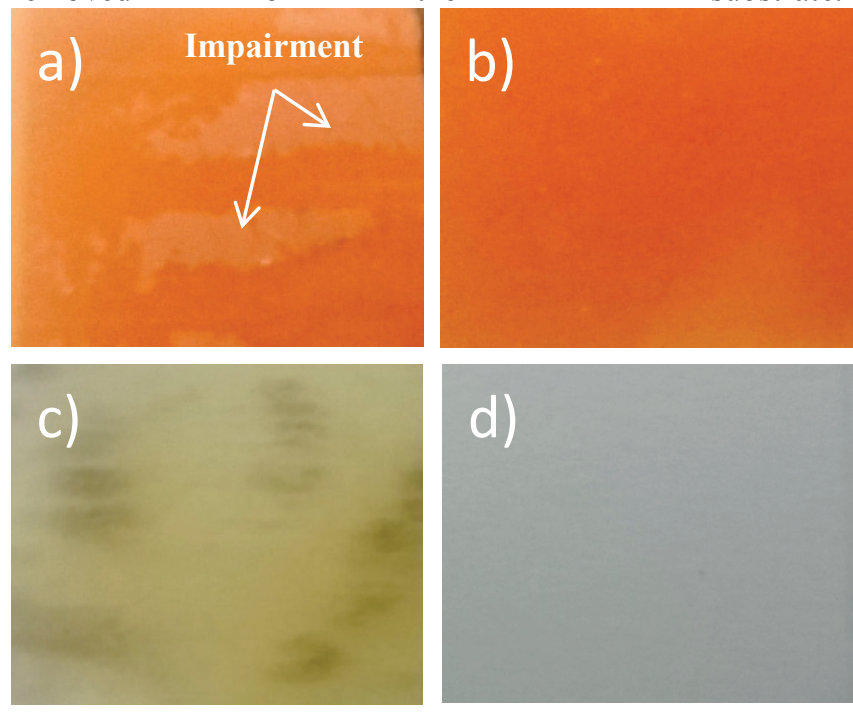

Fig. 8 Top views of various ITO layers deposited on polymer substrates: a) impairments on surface of a consolidated ITO layer on PI substrate due to incomplete removal of organic agents (consolidation fluence approximately $40 \mathrm{~mJ} / \mathrm{cm}^{2}$ ); b) consolidated ITO layer on PI substrate with complete removal of organic agents (consolidation fluence approximately $40 \mathrm{~mJ} / \mathrm{cm}^{2}$ ); c) impairments of ITO coated PP substrate after $\mathrm{CO}_{2}$ laser irradiation; d) ITO coated PP substrate.

To reduce the thermal load of the PP substrates laser irradiation with a shorter optical penetration depth is required. Utilizing laser irradiation with shorter wavelength leads to a reduction of the optical penetration depth. The photon energy of a $\mathrm{KrF}$ excimer laser of $5.0 \mathrm{eV}$ exceeds the band gap of ITO which is between $3.5 \mathrm{eV}$ and $4.0 \mathrm{eV}$. Due to interband interaction the optical penetration depth of ultraviolet laser systems is minimal [17]. For our experiments in the ultraviolet wavelength range we used the $\mathrm{KrF}$ excimer laser mentioned before. In this case the laser pulse is completely absorbed in the first approximately $50 \mathrm{~nm}$ of the deposited nanoparticle ITO layer.

Simulations of the heat distribution caused by single pulse nanosecond UV laser irradiation for $\mathrm{ZnO}$ nanoparticle layer indicate that the porous nanoparticle ITO layer is capable of shielding the underlying organic substrate from excessive heating due to poor heat conductivity of the porous nanoparticle layer and that the thermal relaxation time of the ITO layer is less than the repetition rate of the $\mathrm{KrF}$ excimer laser. The results of the thermal simulation for $\mathrm{ZnO}$ nanoparticle layer are applicable to the thermal behavior of ITO nanoparticle layers, because $\mathrm{ZnO}$ and ITO nanoparticle layers have similar thermal properties. The simulated relaxation time of nanoparticle $\mathrm{ZnO}$ layer is approximately $0.5 \mu \mathrm{s}$. Consequently it can be expected, that the thermal relaxation time of nanoparticle ITO layers is also less than the repetition rate of the $\mathrm{KrF}$ laser. As a result the heating process is independent from the pulse frequency of the excimer laser [15].

However, experiments have shown that the $\mathrm{KrF}$ laser based removal process is also limited. High fluences significantly heat up the surface of the nanoparticle ITO layer which leads to a partial consolidation the particle system. Arbitrary small fluences could not be realized due to limitations in the experimental setup. Figure 9 shows the changes of the relative resistance of various ITO nanoparticle layers deposited on PP substrates at different number of pulses and fluences. The UV laser treatment significantly decreases the sheet resistance of ITO layers deposited on PP substrates as shown in figure 10.
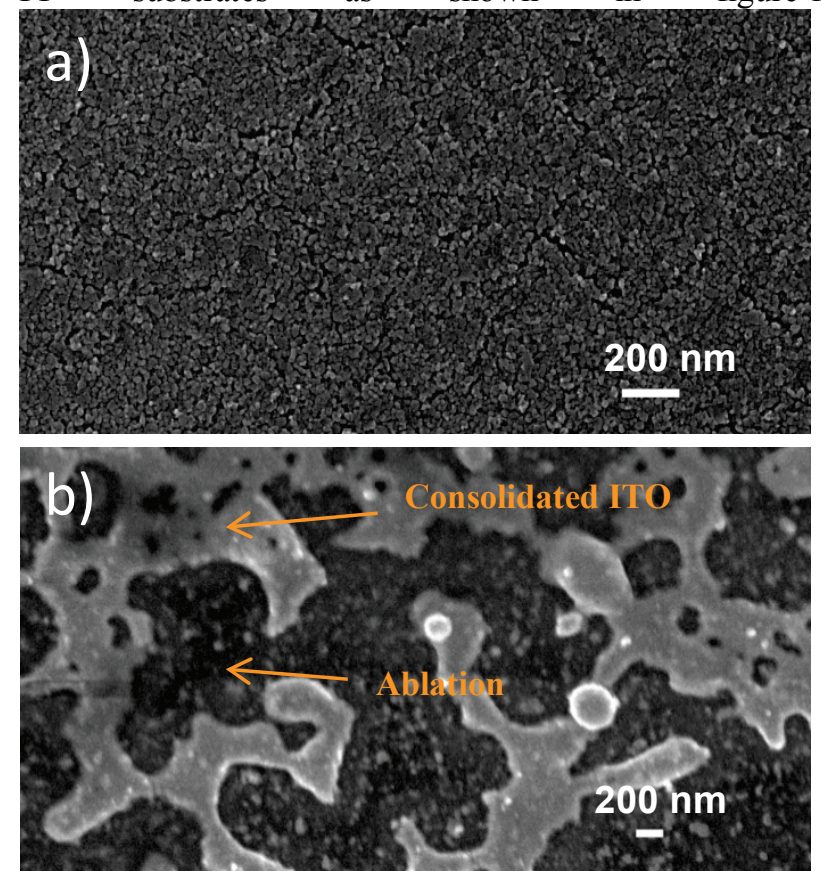

Fig. 9 Top view of a SEM image of a) a ITO layer after removal of organic compounds utilizing excimer laser irradiation (100 pulses at a fluence of $16.7 \mathrm{~mJ} / \mathrm{cm}^{2}$ per pulse) and $b$ ) a consolidated ITO layer (consolidation fluence of approximately $84.4 \mathrm{~mJ} / \mathrm{cm}^{2}$ ) after removal of organic compounds utilizing excimer laser irradiation (100 pulses at a fluence of $16.7 \mathrm{~mJ} / \mathrm{cm}^{2}$ per pulse). Both layers were deposited on soda lime glass substrates.

Best results could be accomplished with 100 laser pulses at a pulse fluence of $16.7 \mathrm{~mJ} / \mathrm{cm}^{2}$. With these parameters the sheet resistance could be reduced to a value of approximately $1.5 \mathrm{M} \Omega$. The decrease of the sheet resistance after $\mathrm{KrF}$ laser irradiation is affiliated to the generation of sinter necks. But the energy deposited in the nanoparticle layer 
was not high enough to cause a complete densification of the surface of the nanoparticle layer. A subsequent consolidation step partly further consolidates and simultaneously ablates the ITO layer which is shown in figure 9.

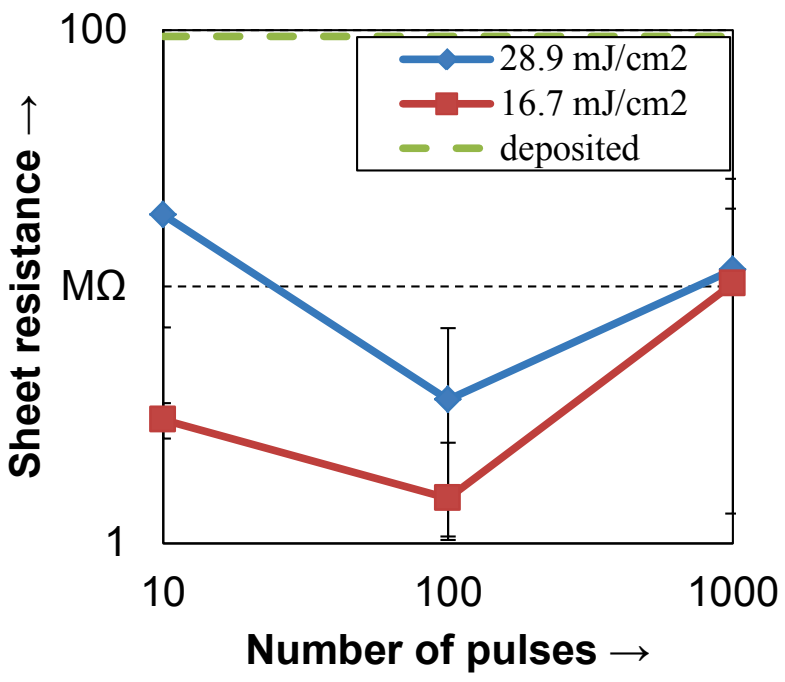

Fig. 10 Sheet resistance of ITO nanoparticle layers deposited on PP substrates (deposited) and after UV irradiation (fluence of approximately $28.9 \mathrm{~mJ} / \mathrm{cm}^{2}$ and $16.7 \mathrm{~mJ} / \mathrm{cm}^{2}$ ).

\section{Conclusion}

In this work we have shown the possibility to remove organic compounds from nanoparticle ITO layers deposited on polymer substrates. For high-temperature polymer substrates, like PI, $\mathrm{CO}_{2}$ laser irradiation has proven ideal for the removal of organic compounds. The high thermal capacity of these polymers prevents them from thermal impairment during the $\mathrm{CO}_{2}$ laser irradiation. However the sheet resistance of layers consolidated on PI substrates is slightly increased compared to those consolidated on glass substrates. Consolidated ITO layers on PI substrates show a higher density of defects compared to consolidated ITO layers deposited on glass substrates because the composite structure generates mechanical tension due to a mismatch in the coefficients of thermal expansion. $\mathrm{CO}_{2}$ laser based removal of organic compounds is not applicable for PP substrates, because the PP exhibits inferior thermal resistivity. Consequently, the PP substrate is damaged by the $\mathrm{CO}_{2}$ laser irradiation and the ITO nanoparticle layer is ablated. Whereas $\mathrm{KrF}$ laser irradiation lead to a significant decrease of the sheet resistance of deposited ITO layer on PP substrates due to a partial densification of the nanoparticle layer surface. A subsequent laser consolidation leads to an inhomogeneous ITO layer structure. Parts of the ITO layer are further densified while other parts of the ITO layer are ablated. Our investigations indicate that infrared laser irradiation is applicable for the generation of optoelectronic devices, like solar cells, displays or disposals, on thermal resistant polymer substrates. The efficiency of the infrared laser based removal process is highly depending on the thermal stability of the polymer substrate.

\section{Acknowledgments}

The authors gratefully thank the German Research Foundation (DFG, Graduiertenkolleg 1161/2). In addition, we are grateful for the support by Evonik Industries AG. Moreover, the authors gratefully acknowledge funding of the Erlangen Graduate School in Advanced Optical Technologies (SAOT) by the German Research Foundation (DFG) in the framework of the German excellence initiative.

\section{References}

[1] T. Maruyama and K. Fukui: Thin Solid Films 203, (1991) 297.

[2] J. George and C. S. Menon: Surf. Coat. Tech. 132, (2000) 45.

[3] T. Minami, H. Sonohara, T. Kakumu and S. Takata: Thin Solid Films 270, (1995) 37.

[4] J. Puetz and M. A. Aegerter: Thin Solid Films 516, (2008) 4495

[5] N. Kölpin, M. Wegener, E. Teuber, S. Polster, L. Frey and A. Roosen: J. Mater. Sci. 48, (2013) 1623.

[6] M. Baum, H. Kim, I. Alexeev, A. Piqué and M. Schmidt: Appl. Phys. A 111, (2013) 799.

[7] J. Puetz and M. A. Aegerter: Thin Solid Films 516, (2008) 4495

[8] F. Kurdesau, G. Khripunov, A.F. da Cunha, M. Kaelin and A.N. Tiwari: J. Non-Cryst. Solids 352, (2006) 1466.

[9] A.K. Kulkarni, K. H. Schulz, T.S. Lim and M. Khan: Thin Solid Films 345, (1999) 273.

[10] N. Outaleb, J. Pinel, M. Drissi and O. Bonnaud: Microw. Opt. Technol. Lett. 24, (2000) 3.

[11] L. Han, N. Koide, Y. Chiba, A. Islam, R. Komiya, N. Fuke, A. Fukui and R. Yamanaka: Appl. Phys. Lett. 86, (2005) 213501.

[12] G. Legeay, X. Castel, R. Benzerga and J. Pinel: phys. stat. sol. (c) 5, (2008) 3248 .

[13] T. Tsuchiya, F. Yamaguchi, I. Morimoto, T. Nakajima and T. Kumagai: Appl. Phys. A 99, (2010) 745.

[14] M. Mahajeri, A. Schneider, M. Baum, T. Rechtenwald, M. Voigt, M. Schmidt and W. Peukert: Thin Solid Films 520, (2012) 5741.

[15]M. Baum, S. Polster, M. P. M. Jank, I. Alexeev, L. Frey and M. Schmidt: JLMN 8, (2013) 144.

[16]S. K. Park, J. I. Han, W. K. Kim and M. G. Kwak: Thin Solid Films 397, (2001) 49.

[17] G. Legeay, X. Castel, R. Benzerga and J. Pinel: phys. stat. sol. (c) 5, (2008) 3248 .

(Received: May 24, 2015, Accepted: February 4, 2016) 